\title{
DISOLUCIÓN MATRIMONIAL DE PAREJAS DEL MISMO SEXO ¿A quién se atribuye la custodia de los hijos?
}

\author{
José Manuel Jimenez Cabello \\ Universidad de Granada, Granada, España \\ Diego Becerril Ruíz \\ Universidad de Granada, Granada, España \\ José Manuel García Moreno \\ Universidad de Málaga, Málaga, España
}

\begin{abstract}
Resumen Tras la puesta en marcha de la Ley 15/2005 son bastantes las parejas del mismo sexo que han contraído matrimonio. Tras 15 años, muchas de ellas se han disuelto, lo que conlleva que otras tantas se hayan visto envueltas en procesos de asignación de custodia de los hijos. El presente artículo pretende conocer cómo se distribuyen las modalidades de custodia tras el divorcio de matrimonios compuestos por cónyuges del mismo sexo y que variables sociales se relacionan con las mismas. Se hace uso de metodología cuantitativa utilizando, concretamente, la Estadística de Nulidades, Separaciones y Divorcios (INE) y aplicando análisis de tipo descriptivo y multivariante. Se concluye que la custodia compartida es una modalidad al alza tras la disolución de estos matrimonios, superando desde 2016 a la modalidad exclusiva. Además, variables como la tenencia de hijos menores o mayores dependientes, la existencia de ley en materia de custodia de cada comunidade autónoma, la edad de los cónyuges y el fallo de la sentencia, son variables que influyen en la modalidad de la misma.
\end{abstract}

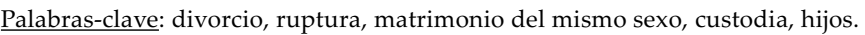

Dissolução matrimonial entre pessoas do mesmo sexo: a quem é atribuída a custódia dos filhos?

Resumo Após a implementação da Lei 15/2005, muitos casais do mesmo sexo casaram-se. Após 15 anos, muitos deles separaram-se, o que levou a que muitos mais casais estivessem envolvidos em processos de custódia de menores. Este artigo visa perceber como os acordos de custódia são distribuídos após o divórcio em casamentos entre pessoas do mesmo sexo e que variáveis sociais os influenciam. É aplicada a metodologia quantitativa, utilizando especificamente as Estatísticas sobre Anulações, Separações e Divórcios (INE) e aplicando análises descritivas e multivariadas. Conclui-se que a custódia partilhada é uma modalidade crescente após a dissolução destes casamentos, ultrapassando desde 2016 a custódia exclusiva. Além disso, variáveis como a existência de filhos menores ou maiores dependentes, a existência de uma lei de custódia em cada comunidade autónoma, a idade dos cônjuges e a decisão da sentença são variáveis que influenciam a modalidade de custódia.

Palabras chave: divórcio, separação, casamento entre pessoas do mesmo sexo, custódia, filhos.

Marriage dissolution of same-sex couples: to whom is custody of the children attributed?

Abstract After the implementation of Law 15/2005, there are quite a few same sex couples who have married. After 15 years, many of them have dissolved, which means that many others have been involved in child custody allocation processes. This article aims to know how custody modalities are distributed after the divorce of same-sex marriages and what social variables influence them. For this, quantitative methodology is applied, using, specifically, the Statistics on Annulments, Separations and Divorces (INE) and applying descriptive and multivariate analyses. It is concluded that shared custody is an upward modality after the dissolution of these marriages, surpassing since 2016 the exclusive modality. In addition, variables such as the possession of dependent minor or elder children, the existence of the custody law of each Autonomous Community, the age of the spouses and the judgment of the sentence, are variables that influence its modality.

Keywords: divorce, rupture, same-sex marriage, custody, children. 
Résumé Après l'entrée en vigueur de la loi 15/2005, de nombreux couples de même sexe ont contracté mariage. Après 15 ans, beaucoup d'entre eux ont été dissous, ce qui signifie que beaucoup d'autres ont été impliqués dans des processus d'attribution de la garde des enfants. Cet article vise à savoir comment se répartissent les modalités de garde après le divorce dans les mariages composés de conjoints de même sexe et quelles variables sociales leur sont liées. Une méthodologie quantitative est utilisée, en particulier en utilisant les statistiques de nullité, de séparation et de divorce (INE) et en appliquant une analyse descriptive et multivariée. Il est conclu que la garde partagée est une modalité en augmentation après la dissolution de ces mariages, dépassant la modalité exclusive depuis 2016. En outre, des variables telles que la possession d'enfants mineurs ou âgés à charge, l'existence d'une loi sur la garde de chaque communauté autonome, l'âge des conjoints et la décision de justice, sont des variables qui influencent la modalité de celle-ci.

Mots clefs: divorce, rupture, mariage homosexuel, garde, enfants.

\section{Introducción}

La sociedad europea ha vivido, en las últimas décadas, un cambio social sin precedentes, viéndose afectados diversos ámbitos de la realidad social, como el laboral, el educativo o la familia, entre otros. El interés, desde una perspectiva sociológica, por ver las implicaciones de estos cambios en el ámbito familiar ha ido creciendo con el paso del tiempo encontrando extensa literatura (Wall y Gouveia, 2014; Morgan, 2011; Widmer, 2010; Déchaux, 2009; Sánchez y Bote, 2009; Finch, 2007). Mayor protagonismo y consolidación de nuevas formas familiares, aumento de la esperanza de vida, la importancia de las relaciones intergeneracionales, cambios en la nupcialidad y natalidad o redefinición de los roles de género son algunos aspectos a destacar (Juárez, 2007). España no ha permanecido exenta a estas transformaciones producidas en el entorno familiar, destacando dos aspectos sobremanera: la ruptura matrimonial, y su percepción social, y la legalización del matrimonio entre personas del mismo sexo.

En primer término, el cambio en la percepción de la ruptura matrimonial ha quedado reflejada, en cierta manera, en los cambios ocurridos a nivel legislativo (Becerril, 2008). Destaca la introducción de la Ley 15/2005, de 8 de julio, por la que se modifican el Código Civil y la Ley de Enjuiciamiento Civil en materia de separación y divorcio. Ésta facilitaba el divorcio tanto desde el punto de vista burocrático como temporal. Además, esta regulación introducía un aspecto novedoso en lo que refiere a una de las principales consecuencias derivadas del proceso de divorcio cuando se tienen hijos o hijas: incluía en el ordenamiento jurídico, por primera vez, la posibilidad de otorgar la modalidad de custodia compartida, algo que ya existía en el entorno europeo en países como Alemania (1997), Austria (2001) o Francia (2002), por ejemplo (Jiménez, Becerril y García, 2020). Esta nueva situación condujo a que pocos años después de la aprobación de esta ley (desde 2010) en distintas comunidades autónomas (CA en adelante), ${ }^{1}$ como Aragón,

$1 \quad$ Entidades territoriales que, dentro del ordenamiento jurídico constitucional español, poseen autonomía, con instituciones y representantes propios y determinadas competencias legislativas, ejecutivas y administrativas. 
Cataluña o Comunidad Valenciana, se comenzara a legislar sobre las situaciones y las relaciones con los menores tras el divorcio (Solsona, Spijker y Ajenjo, 2017; Jiménez, Becerril y García, 2020).

En segundo término, otro aspecto novedoso fue el que supuso la reforma del Código Civil en 2005, que incorporó en el ordenamiento jurídico español la legalización del matrimonio entre personas del mismo sexo, convirtiendo a España en el tercer país del mundo que legalizaba estas uniones (Cortina y Cabré, 2010). Dicha ley dio respuesta a un proceso complejo, con multitud de demandas y situaciones diversas (Pichardo, 2004). Señalar, que el proceso de aprobación de las uniones entre personas del mismo sexo en el contexto europeo se inicia durante la década de 1990, concretándose de forma distinta en cada país. La diversidad internacional se ve reflejada en los diferentes contenidos de las normativas legales y los factores contextuales, que se fundamenta sobre todo en la aceptación e integración de este tipo de parejas en la sociedad (Martín, 2016). En este sentido, Países Bajos y Bélgica fueron los primeros en legalizar este tipo de matrimonios. Posteriormente, otros tantos países europeos comenzaron a legislar en torno a esta cuestión: España (2005), Suecia (2009), Portugal (2010), Francia (2013) o Italia (2016), entre otros.

Sin embargo, cabe destacar que la ley impulsada en España fue novedosa a nivel mundial por ser la primera que reconocía el matrimonio entre personas del mismo sexo a través de la reforma del Código Civil, y no mediante la aprobación de una nueva figura jurídica (Martín, 2016). Este cambio normativo es sumamente importante, ya que no solo se comenzó a legislar el matrimonio entre personas del mismo sexo, sino que trajo consigo aspectos derivados como las posibles rupturas de este tipo de matrimonios y la posibilidad de tenencia/adopción de hijos.

Estos dos grandes cambios, impulsados en 2005, dados en la esfera familiar, van de la mano en cuanto al objeto de estudio de la presente investigación: la asignación de la custodia de hijos e hijas tras la disolución de matrimonios compuestos por personas del mismo sexo.

La asignación de la custodia de hijos e hijas tras la ruptura matrimonial se ha ido posicionando como una cuestión sociopolítica central, que se traduce en un mayor debate en la actualidad. A nivel estatal, la falta de acuerdo ha impedido que incluso los anteproyectos de ley presentados se hayan tramitado. En la etapa de Alberto Ruíz Gallardón como ministro de Justicia (2011-2014), se planteó la posibilidad de regular la custodia incluyendo, de alguna forma, la modalidad compartida como más recurrente de lo que constituye en la actualidad, aunque finalmente no se llegó a realizar. De la misma forma, Rafael Catalá, también ministro de Justicia (2014-2018), se comprometió a presentar un proyecto de ley de corresponsabilidad parental mediante el que se regulara la custodia compartida que no llegó a concretarse al finalizar la legislatura. De hecho, y con respecto a los divorcios de matrimonios heterosexuales, esta cuestión ha tenido continuo seguimiento científico en España existiendo vasta literatura (Jiménez y Becerril, 2020; Solsona, Spijker y Ajenjo, 2017, Becerril 2014; Flaquer, 2012, entre otros).

En el caso concreto de las disoluciones de matrimonios compuestos por cónyuges del mismo sexo, un porcentaje muy importante lo hace con la presencia de hijos e hijas (según la Estadística de Nulidades, Separaciones y Divorcios - ENSD 
en adelante - un 39,5 en 2017), por lo que la cuestión no es baladí. Además, en torno a esta realidad social cada vez más presente se visualiza la escasa investigación llevada a cabo, y sobre todo desde una óptica sociológica, en España. Son precisamente estos aspectos los que conducen a establecer como objetivo de esta investigación conocer cuáles son las variables de carácter sociológico que puedan incidir en la asignación de la custodia a favor de un determinado modelo tras el divorcio de matrimonios del mismo sexo.

\section{Investigaciones referidas a parejas del mismo sexo}

Si se procede a realizar una revisión de la literatura existente en torno a la disolución de matrimonios de personas del mismo sexo, y los procesos de custodia derivados de éstos, se advierte una importante falta de estudios empíricos, desde una perspectiva sociológica, en España. Esto no quiere decir que la atención sobre este grupo no haya sido amplia, si bien se ha centrado en otros ámbitos como el derecho (Fernández, 2006; Martín, 2016).

En este sentido existen investigaciones, siendo bastante diversas, que hacen referencia a la evolución del matrimonio compuesto por personas del mismo sexo y a las características de la pareja. Destacan los estudios de Meil $(2001,2003)$, donde se investigó a las uniones de hecho incluyendo este tipo de parejas. Por otro lado, se pueden señalar los análisis realizados por Cortina y Cabré (2010) y Esteve, Cortina y Cabré (2009). En ellos se señala, por un lado una alta concentración de parejas del mismo sexo en zonas urbanas, con unas edades de los miembros más jóvenes que en el caso de las parejas heterosexuales. Por otro lado, se desprende que se produce una unión de forma homógama de acuerdo a variables como la edad y nivel educativo. Cortina (2016) apunta a un perfil de este tipo de parejas marcadamente heterógamo y a un predominio del modelo igualitario de pareja. Más actual es el trabajo elaborado por Capote y Nieto (2018), en el que se analizan aspectos como la propia evolución de los matrimonios, su distribución geográfica e incluso se trazan los perfiles de los miembros de este tipo de parejas.

Por otra parte, se observan estudios que se encuadran en el ámbito del derecho o del análisis de la propia ley en el colectivo homosexual. Por ejemplo, Calvo (2010) hace referencia a la relación entre los movimientos sociales y el reconocimiento de los derechos civiles, centrándose en la legalización del matrimonio entre personas del mismo sexo en España. Fernández (2006), por su parte, presenta la evolución jurídica del sistema matrimonial español desde 1978 hasta la aparición del matrimonio entre personas del mismo sexo, mientras que en el trabajo de Martín (2016) se muestra una comparación de las realidades legales de este tipo de uniones en Europa. Por último, Gimeno y Barrientos (2009) hacen un repaso sobre la institución matrimonial tras la legalización del matrimonio homosexual.

Sin embargo, y como se ha citado anteriormente, es más complicado encontrar investigaciones acerca de la disolución de estas parejas y sus características a nivel nacional. Becerril y Jiménez (2016) realizaron un primer acercamiento, presentando un breve análisis sobre el grado de consenso o disenso existente en 
divorcios de matrimonios del mismo sexo. En él afirmaron la existencia de una tendencia al alza de la ruptura de estos matrimonios. También se destaca, someramente, que suele existir un porcentaje más alto de consenso en los divorcios de este tipo de matrimonio que en los heterosexuales.

En el plano internacional la cobertura sobre esta tipología de parejas ha sido bastante amplia. Se observan numerosas investigaciones que pueden encuadrarse en dos ámbitos principales: características sociodemográficas de estas parejas y como son sus disoluciones. En el primer grupo, destacan diversos estudios. Remiggi (1998) se centra en conocer las zonas donde habitan, con más frecuencia, este tipo de parejas y el por qué. Por otro lado, Black et al. $(2000,2002)$ hacen referencia a la concentración en las ciudades de este tipo de parejas. Andersson et al. (2006), por su parte, concluyen como las parejas noruegas y suecas son más dispares en cuanto a su nivel educativo. A esta conclusión se llegó también en el caso francés, de la mano de Festy, Digoix y Garnier (2004). De nuevo el eje rural-urbano ofrecería información imprescindible para comprender este fenómeno, en este caso, a través del estudio de Leroy (2006). En este, se exploran las bondades y aspectos positivos de la ciudad que generan una suerte de atracción hacia ella, destacando la mayor posibilidad de encontrar pareja. Por último Giraud (2013) centra su preocupación de nuevo en las zonas donde habitan este tipo de parejas y las razones que empujan a ello.

En cuanto a las disoluciones de estas parejas y matrimonios, sí se observan en el ámbito internacional más investigaciones que conducen a conocer las principales características de estas. El análisis de Blumstein y Schwartz (1983), que más tarde continuo Kurdek (1991, 1992), destaca entre las principales conclusiones que las parejas del mismo sexo tienen mayor tasa de ruptura. Esta además, es doble en el caso de las lesbianas que en los gays, interesando, sobre todo, que en determinadas investigaciones se detecta el bajo nivel de conflictividad en las disoluciones de parejas compuestas por personas del mismo sexo (Goldberg y Allen, 2013). Esto conduce a que la mayor parte de las custodias de hijos e hijas sean compartidas (Gartrell et al., 2011). De forma más reciente, se concluye que las parejas masculinas permanecen juntas más tiempo que las parejas heterosexuales o lesbianas, teniendo estas últimas 1,5 veces más probabilidades de ruptura que las parejas heterosexuales (Rothblum, Bálsamo y Wickham, 2018). Estos autores examinaron a 512 parejas de Vermont durante un periodo temporal de 12 años, prestando atención a variables como la duración de la relación, el nivel educativo y la renta o tenencia de hijos, entre otras.

Otras investigaciones a destacar son aquellas centradas en la tenencia de hijos e hijas por parte de este tipo de matrimonios (Gato, Rangel y Leal, 2020) e incluso aquellas que abordan las habilidades parentales de este tipo de parejas (Gato, Freitas y Fontaine, 2012). De tal forma, se constata que, a nivel internacional, el estudio de las rupturas de parejas y matrimonios formados por personas del mismo sexo si ha tenido seguimiento continuo, así como las principales características de estas. Sin embargo, queda patente la falta de investigaciones empíricas en España acerca de las disoluciones de este tipo de matrimonios y, sobre todo, de la asignación de la custodia si hay hijos/as presentes. 


\section{Evolución de la disolución de matrimonios compuestos por personas} del mismo sexo y tenencia o no de hijos e hijas

Para poder abordar los procesos de custodia tras la disolución de este tipo de matrimonios en España, es condición sine qua non conocer la evolución de los divorcios. Esto es necesario ante la importante falta de seguimiento a esta cuestión. La evolución de los divorcios entre parejas del mismo sexo se ha mantenido, relativamente, estable en el tiempo (figura 1).

En 2007 las disoluciones de matrimonios conformados por varones (42) fueron más del doble que las producidas en los compuestos por mujeres (17), que se situaban en su cifra más baja. De forma conjunta, los divorcios de ambos tipos de matrimonio aumentan hasta el año 2015. A partir de este año se observan dos dinámicas semejantes en las que destacan los divorcios entre varones. Las disoluciones de matrimonios compuestos por hombres sufren un fuerte subida (de 2015 a 2016 aumentan en 331) alcanzando su nivel más alto de divorcios en 2016 (820) mientras que las producidas en matrimonios compuestos por mujeres se mantenían en cifras parecidas aumentando con respecto a 2015 en 115, llegando a su valor más elevado en 2016 (503). Esto, puede estar relacionado con el hecho de que el stock de varones que se han ido casando desde 2007 ha sido bastante más alto que el de mujeres hasta 2014. Es a partir de esta fecha cuando la dinámica se iguala, alcanzado los dos tipos de matrimonios del mismo sexo, prácticamente, idénticos niveles (1596 entre mujeres por 1679 de varones). Del total de matrimonios producidos entre varones (21.902) se han divorciado, hasta 2017, un total de 3407 (15,6\%) mientras que los contraídos entre mujeres (16.796) se han disuelto 2526 (13,03\%).

Cuestión derivada e interesante es conocer cómo se producen estas rupturas y que factores pueden hacer el proceso más o menos complejo. En este caso, la tenencia o no de hijos parece un elemento clave. Suso et al. (2012) hacen referencia a que cuando las parejas cuentan con hijos la situación se vuelve más compleja y la solución a la custodia de los menores constituye un proceso con múltiples aristas sobre el que sigue debatiendo y reflexionando en la actualidad. De la tenencia o no de hijos depende, en gran medida, un proceso negociador bastante complejo como es la asignación de la custodia.

Apreciando la figura 2, se puede ver que la tenencia de hijos e hijas por parte de este tipo de matrimonios es frecuente. Especificando aún más, en el caso de disolución matrimonial, siguen suponiendo un porcentaje importante aquellas parejas que se disuelven con hijos, llegando a alcanzar el 39,5\% de las mismas en 2017.

Si bien es cierto que la cantidad, año a año, no es amplia, el total de disoluciones matrimoniales entre personas del mismo sexo que tienen hijos e hijas es importante, más aun teniendo en cuenta que se está ante una población que ha ido aumentando de forma continuada desde la legalización de este tipo de matrimonio, lo que ha provocado la subida, también, del número de disoluciones.

Ciertamente es destacable como la disolución de este tipo de matrimonios y sus características no han tenido un seguimiento continuado o intenso para el caso de España, país pionero en regular el matrimonio homosexual (Becerril y Jiménez, 2016). Ni siquiera, un mero acercamiento empírico. En este sentido, no se puede 


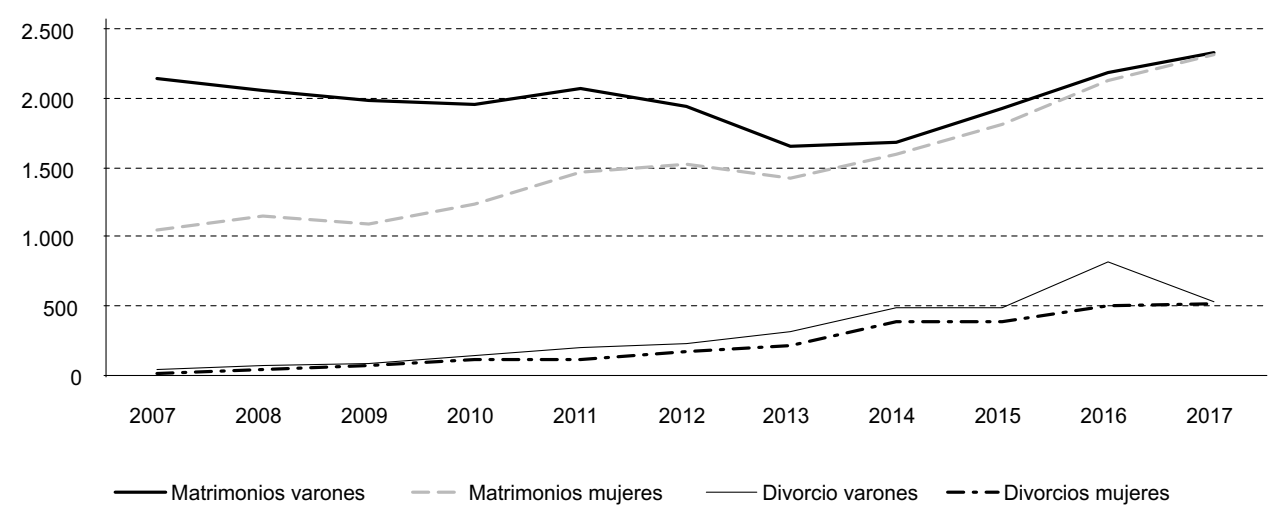

Figura 1 Evolución de la ruptura de matrimonios de personas del mismo sexo en España (2007-2017) (miles)

Fuente: elaboración propia a partir de datos de la Estadística de Nulidades, Separaciones y Divorcios.

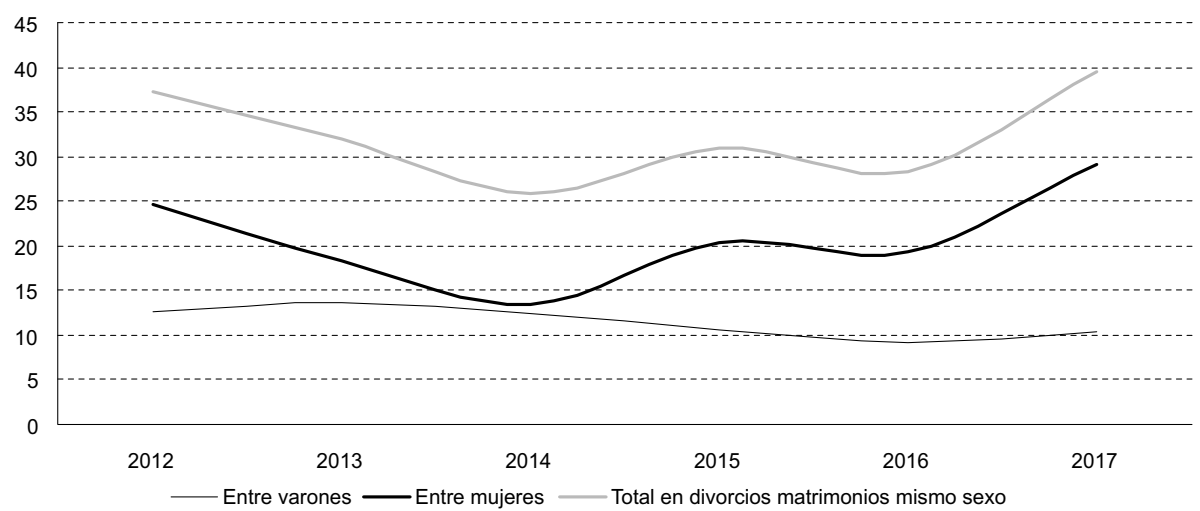

Figura 2 Hijos presentes en disoluciones de matrimonios de personas del mismo sexo según su composición en España (2012-2017) (\%)

Nota: precisar que únicamente se pueden utilizar datos desde el año 2012 debido a que tras la puesta en marcha de la ENSD por parte del Instituto Nacional de Estadística, no se incluyen datos referentes a la tenencia de hijos, tipo de proceso o asignación de la custodia para los matrimonios entre personas del mismo sexo hasta 2012.

Fuente: elaboración propia a partir de datos de la Estadística de Nulidades, Separaciones y Divorcios.

aludir a la falta de datos, ya que la ENSD permite acceder al número de rupturas y algunas de sus características principales. Además, existe ya un periodo temporal que permite que se obtenga una cantidad de datos mínima a poder analizar. 


\section{La asignación de la custodia tras la disolución matrimonial}

Cuando se aborda la cuestión relativa a la asignación de custodias tras el divorcio de matrimonios del mismo sexo, rápidamente se observa la falta de investigaciones relativas a esta población. Sin embargo si existen investigaciones muy completas a nivel nacional e internacional sobre la asignación de la custodia tras la ruptura de matrimonios heterosexuales. En el primer ámbito del seguimiento de este fenómeno social se observan investigaciones bastante completas. Destacar las realizadas por Solsona, Spijker y Ajenjo (2017), Becerril (2014, 2015), Jiménez y Becerril (2020), Flaquer (2012) o Ruíz y Alcázar (2017), en las que han comenzado a analizar las variables sociales que pueden ser determinantes en la asignación de la custodia de los hijos. Aun así, es preciso destacar que este seguimiento no ha sido continuo ni muy intenso, circunscribiéndose a pocos estudios.

En el ámbito internacional el seguimiento realizado sobre esta cuestión permite, incluso, dividir por conjuntos de variables (edad y nacionalidad de los cónyuges, número de hijos menores, fallo de la sentencia, etc.). En este sentido, atendiendo a las características de la pareja, se puede hacer referencia a determinadas variables que pueden influir en la asignación de la custodia. Estas son: la tenencia de hijos menores o mayores dependientes y el número de estos, la nacionalidad de los cónyuges, el fallo de la sentencia o la edad de los cónyuges.

La duración de la relación, según Yeung et al. (2001), es importante porque la misma puede estar ligada a la edad de los hijos, siendo un elemento fundamental en la asignación de la custodia, ya que no es lo mismo que el hijo custodiable sea un recién nacido a que este tenga una edad más avanzada. Además, se señala como la probabilidad para que los padres obtengan la custodia aumenta a medida que los hijos crecen.

En cuanto al número de hijos, igualmente puede afirmarse que el número de los mismos, e incluso que estos sean menores o mayores dependientes, puede influir en la asignación de la custodia, como ya indicó en este sentido Becerril (2015: 62): "la custodia compartida aparece con más fuerza en aquellas situaciones con dos o más hijos siendo menos frecuente con uno solo". Además, para reafirmar esta idea, puede ser útil el estudio llevado a cabo por Cancian y Meyer (1998), en el que se refleja que en el posible caso de que existan varios hijos tras del proceso de divorcio, los padres están de acuerdo en no separar a los hermanos en el $97 \%$ casos. Así, en sólo el $3 \%$ de los juicios con varios hijos menores de edad y con el acuerdo los padres, algunos niños viven con su madre, y el otro con su padre. Por el contrario, Maccoby y Mnookin (1992) indican que la compartida es más probable en parejas con un solo hijo.

Estos mismos estudios, mostraron que la custodia para el padre es más probable entre los niños mayores. Mientras que Cancian y Meyer (1998) no encontraron ninguna relación determinante con la edad de los niños, Maccoby y Mnookin (1992) observaron que los niños con edades comprendidas entre 2 y 9 años de edad tienen más probabilidades de tener un régimen de custodia compartida que exclusiva. Es preciso recordar que estas investigaciones se centraban, de forma exclusiva, en disoluciones de matrimonios heterosexuales. 
En cuanto a la nacionalidad de los cónyuges, Becerril y Jiménez (2016), mediante un estudio realizado para comprobar la conflictividad en las rupturas de matrimonios con cónyuges del mismo sexo en España, comprobaron que es una variable que en ese caso resultaba importante.

Por otro, cómo se desarrolle y finalice el proceso de ruptura es una variable que parece decisiva, ya que en gran medida refleja la más que posible relación entre cónyuges y como pueda ser esta en un futuro. Esto es algo que algunos autores ya han intentado comprobar en otros países. Por ejemplo, según Wilcox, Wolchik y Braver (1998), la custodia compartida es más probable cuando se experimentan baja conflictividad entre los miembros de la pareja.

De esta afirmación se desprende, por tanto, una importante hipótesis de estudio: que en los procesos consensuados en los que se supone un menor grado de conflictividad existirá un mayor porcentaje de custodia compartida en comparación con la exclusiva. Además, Becerril y Jiménez (2016), encuentran como en los matrimonios homosexuales según se haya producido la ruptura (consensuada o contenciosa), la custodia de los hijos puede variar. Otro elemento a destacar es la edad de los miembros de la pareja. Cancian y Meyer (1998) y Juby, Le Bourdais y Marcil-Gratton (2005) indican que en cuanto a la edad de las madres, la tendencia general observada es que la probabilidad de que exista una custodia compartida es alta en madres muy jóvenes, descendiendo en las madres veinteañeras y en los primeros años de la treintena, para posteriormente, ascender de los 33 años en adelante.

De tal forma, y tras revisar como estas variables tienen importancia y determinan, de distinta forma, la modalidad de custodia que se asigna, resultaría interesante proceder a su extrapolación en esta investigación y comprobar si puede o no ser un elemento que incida sobre la custodia tras el divorcio de matrimonios conformados por personas del mismo sexo. Es precisamente la vasta literatura existente para el caso de heterosexuales, lo que hace que extrapolar esta amalgama de variables al análisis de este tipo de matrimonios genere ciertas garantías. Si bien, se está presente ante parejas cuya composición es diferente, el amplio conjunto de variables a analizar si puede permitir reconocer, a priori, que algunas de ellas expliquen en cierta medida la asignación de un determinado modelo. Además, es necesario remarcar que se trata del primer análisis de asignación de la custodia tras el divorcio de matrimonios compuesto por cónyuges del mismo sexo que se realiza para el caso de España, por lo que se entiende necesario comenzar sobre una base empírica ya sólida. Esta investigación puede suponer el primer acercamiento a una realidad social emergente y no analizada.

Por tanto, se establece como objetivo general de esta investigación conocer qué tipo de modalidad de custodia se asigna de forma más frecuente tras la disolución de matrimonios compuesto por personas del mismo sexo y que variables sociales influyen en la misma. Como hipótesis se erigen: en primer lugar que la custodia compartida será la modalidad más asignada y, en segundo, que el fallo de la sentencia, la tenencia de hijos menores o mayores dependientes, la edad de los cónyuges y la CA influyen en la modalidad que se asigna. 


\section{Método}

El método utilizado es de carácter cuantitativo. Concretamente, se utiliza la Estadística de Nulidades, Separaciones y Divorcios (ENSD), proporcionada por el Instituto Nacional de Estadística. La misma recoge datos referentes a las nulidades, separaciones y divorcios que se producen anualmente en España, incluyendo ítems como la edad de los cónyuges, número de hijos, nacionalidad, existencia de pensiones, tipo de custodia, etc.

La limitación de la fuente es que el dato específico de asignación de custodias, para este tipo de matrimonios, se recoge únicamente desde 2012. Esto hace inviable la elaboración de una serie más amplia. No obstante, dado que las transformaciones más significativas son de la última década y media, sí permite obtener un periodo relativamente extenso para el análisis. El último año disponible de esta estadística, y sobre el que se realiza el análisis multivariante, es $2017 .{ }^{2}$

En cuanto al análisis de datos utilizados para este estudio se realiza, en un primer lugar, un análisis descriptivo para poder ofrecer al lector la información de la forma más detallada posible. Asimismo se emplea la técnica multivariante del análisis factorial, técnica de reducción de datos que pretende pasar de un número elevado de variables, a un número más pequeño de elementos explicativos, los factores, que permitan explicar de una manera más sencilla la realidad (Ferrán, 1996). Esta técnica permitirá ganar facilidad para interpretar la realidad al menor coste posible en términos de pérdida de información, condición esencial para seleccionar una técnica de análisis. Para determinar que este análisis es el adecuado, se emplean tanto el test de esfericidad de Barlett (que permite detectar la presencia de relación entre variables) como la medida KMO de adecuación de la muestra. Ya que este análisis pretende erigirse como un primer acercamiento a las variables que influyen en la modalidad de custodia que se asigna, la utilización de esta técnica es apropiada por qué permite conocer que variables independientes son esenciales para explicar la variable dependiente.

Para calcular la matriz de correlaciones se ha utilizado el coeficiente de correlación de Pearson, aunque hay que advertir diferentes opiniones al respecto. Si bien es cierto que el análisis multivariante presenta dificultades a la hora de analizar variables dicotómicas, la necesidad de reducción de datos es una realidad apremiante en ciencias sociales. Autores como Kubinger (2003) señalan la necesidad de aplicar la correlación tetracórica, función no disponible en SPSS, en vez de la de Pearson, ya que da resultados más óptimos en cuanto a las magnitudes de las estimaciones (Hoffman et al., 2013). No obstante, investigadores como Bartholomew (1980) no consideran la utilización del coeficiente de correlación de Pearson como un elemento tan problemático ya que las estimaciones obtenidas a partir de variables con distribución asimétrica no son severamente alteradas si los valores del índice de ajuste root mean square error (RMSEA) se mantienen dentro de los estándares aceptados (0-0,08) (Jöreskog, 2001).

2 Que el análisis de datos se centre en 2017 se sustenta, principalmente, en que tras aplicar el mismo análisis para cada una de las bases de datos (desde 2012 a 2017) los resultados obtenidos son similares y, por ello, se presentan los correspondientes al último año con el fin de mostrar al lector los datos más recientes posibles. 
Tabla 1 Variables utilizadas

\begin{tabular}{|c|c|}
\hline Variables & Valores \\
\hline \multirow{2}{*}{ Tipo de custodia } & 1 - Modalidad exclusiva \\
\hline & 2 - Modalidad compartida \\
\hline \multirow{2}{*}{ Tenencia de hijos menores } & 0 -no \\
\hline & 1,2,3 o más- Si \\
\hline \multirow{2}{*}{ Tenencia de hijos mayores dependientes } & $0-$ no \\
\hline & $1,2,3$ o más- $\mathrm{Si}$ \\
\hline $\begin{array}{l}\text { Comunidad Autónoma } \\
\text { (existencia de ley propia o no) }\end{array}$ & $\begin{array}{l}\text { Andalucía, Aragón, Asturias, I. Baleares, Canarias, } \\
\text { Cantabria, Castilla y León, Castilla - La Mancha, Cataluña, } \\
\text { C. Valenciana, Extremadura, Galicia, Madrid, Murcia, Navarra, } \\
\text { País Vasco, La Rioja, Ceuta, Melilla. }\end{array}$ \\
\hline \multirow{2}{*}{ Fallo de la sentencia } & 1 - Consensuado \\
\hline & 2 - No consensuado \\
\hline \multirow{2}{*}{ Nacionalidad 1er cónyuge } & 1 - Española \\
\hline & 2 - Extranjera \\
\hline \multirow{2}{*}{ Nacionalidad 2do cónyuge } & 1 - Española \\
\hline & 2 - Extranjera \\
\hline Edad 1er cónyuge & 18 hasta +65 \\
\hline Edad 2do cónyuge & 18 hasta +65 \\
\hline \multirow{2}{*}{ Demandante del proceso } & 1 - Primer cónyuge \\
\hline & 2 - Segundo cónyuge \\
\hline
\end{tabular}

Fuente: elaboración propia a partir de la ENSD.

El método utilizado para extraer los factores es el de componentes principales. Este permite resumir la mayor parte de la información inicial en el menor número de factores posibles. Por último, normalmente es difícil interpretar los factores iniciales, por lo tanto, la extracción inicial se rota con la finalidad de lograr una solución que facilite la interpretación (Montoya, 2007). Ante esto, se selecciona la rotación Varimax, que permite mantener la interdependencia entre los factores rotados. Las variables seleccionadas pueden observarse en la tabla 1.

Por último, para poder analizar la influencia del número de hijos menores y mayores dependientes en la asignación de la custodia, se utiliza la técnica del análisis de correspondencias. Precisar que todas las operaciones estadísticas se han realizado con el uso del software estadístico SPSS.25.

\section{Resultados y discusión}

A continuación, se procede a presentar los principales resultados obtenidos. En primer lugar se aborda el total de custodias asignadas tras la disolución de estos matrimonios. Como se indicó con anterioridad la serie que se puede elaborar es, únicamente, aquella que va desde el año 2012 hasta la actualidad. 


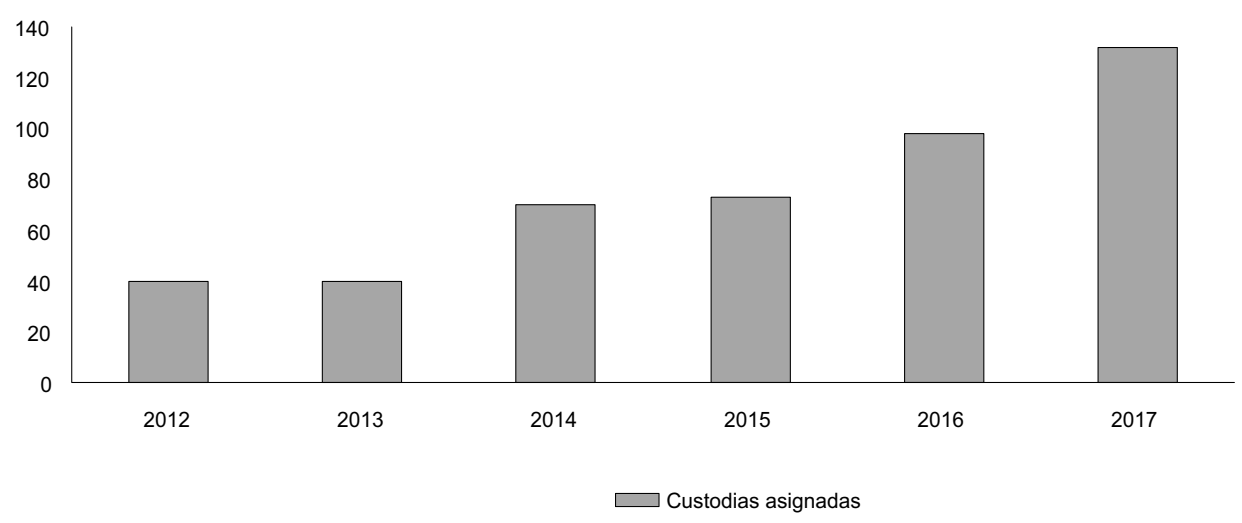

Figura 3 Total de procesos de asignación de custodia tras el divorcio de matrimonios de persona del mismo sexo en España (2012-2017).

Fuente: elaboración propia a partir de datos de la Estadística de Nulidades, Separaciones y Divorcios.

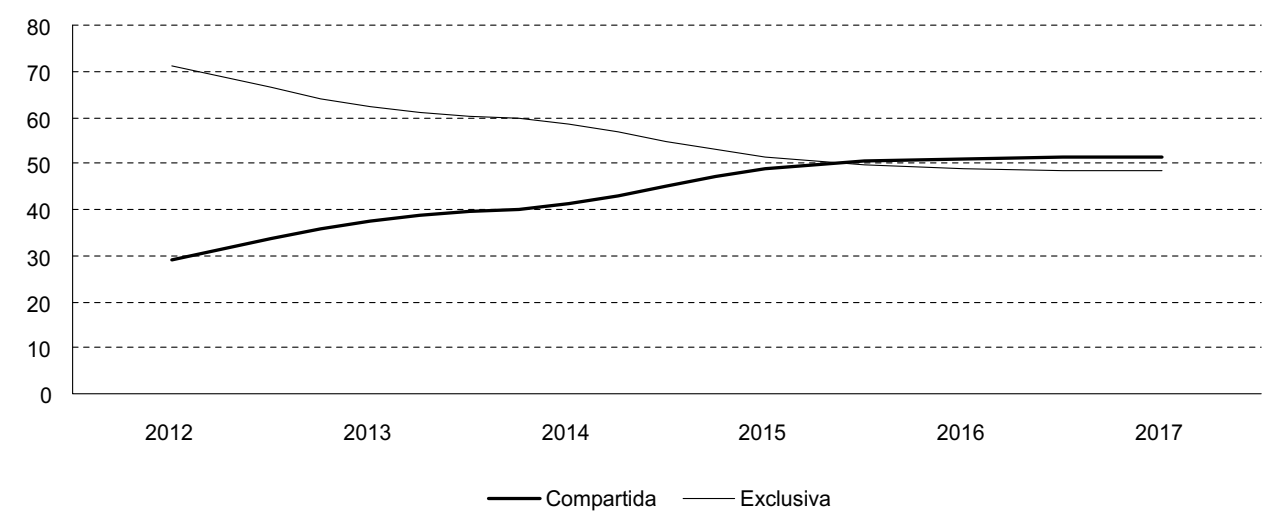

Figura 4 Evolución de la modalidad de custodia asignada tras la disolución de matrimonios de personas del mismo sexo en España (2012-2017) (\%)

Fuente: elaboración propia a partir de datos de la Estadística de Nulidades, Separaciones y Divorcios.

El total de procedimientos sobre asignación de la custodia, en el caso de disoluciones de matrimonios del mismo sexo, ha ido aumentando de forma significativa (figura 3). Concretamente, desde 2012 hasta 2017, se triplican los procesos de divorcio con custodias por asignar. Este aumento puede explicarse debido a que, con el paso del tiempo, son más los matrimonios del mismo sexo que se han ido disolviendo y, por tanto, ha aumentado también el de aquellos que lo hacen con tenencia de hijos/as. En este sentido, es de esperar que con el tiempo en los divorcios de este tipo de matrimonios la presencia de hijos e hijas siga aumentando. Cuestión derivada es conocer a que modalidad pertenecen estas custodias: exclusiva o compartida. 


\begin{tabular}{lcc}
\hline Medida Kaiser-Meyer-Olkin de adecuación de muestreo & 0,504 \\
\hline Prueba de esfericidad de Bartlett & Aprox. Chi-cuadrado & 1860,250 \\
& Gl & 15 \\
& Sig. & 0,000 \\
\hline
\end{tabular}

Fuente: elaboración propia a partir de datos de la Estadística de Nulidades, Separaciones y Divorcios (2017).

La modalidad de custodia compartida ha ido aumentando año tras año en detrimento de las modalidades exclusivas (figura 4). Si bien el año de partida, 2012, presenta la mayor diferencia entre ambas modalidades (70\% de exclusiva por $30 \%$ la compartida), la tendencia hacia el acercamiento de estas es un hecho, suponiendo el año 2016 el punto de ruptura o inflexión. Es en este momento cuando la modalidad compartida supera a la exclusiva (51,0 compartida por 48,9 la exclusiva) confirmándose esta tendencia, de forma clarividente, en 2017 donde la compartida alcanza el 51,5. De esta forma se afirma que la hipótesis I "la custodia compartida será la modalidad más asignada" queda validada. Con esto, es interesante averiguar que variables influyen en la determinación del tipo de custodia que se asigna tras el divorcio de matrimonios formados por personas del mismo sexo.

Atendiendo al análisis factorial realizado $y$, concretamente, a la matriz de componentes rotados, la tenencia de hijos menores o hijos mayores dependientes y el número de estos, el fallo de la sentencia, la edad de los miembros de la pareja y la CA. aparecen como variables a tener en cuenta para explicar la modalidad de custodia que se asigna. Cabe destacar que el estadístico KMO y la prueba de Bartlett (tabla 2) indican la existencia de adecuación de la muestra ya que el estadístico KMO, que varía entre 0 y 1 , arroja un resultado de 0,504 . En este caso no se está presente ante un valor pequeño, por lo que se evita el error de que las correlaciones entre los pares de variables no puedan ser explicadas por otras variables. Como medida, suele establecerse que los valores menores de 0,5 indican que no debe utilizarse el análisis factorial con los datos muestrales que se utilicen (De la Fuente, 2011).

En cuanto a la varianza, atendiendo al cuadro de la varianza total explicada de cada componente y cuáles son los que han sido extraídos, se puede afirmar que los cuatro componentes extraídos acumulan el $61,45 \%$ de la variabilidad de las variables originales. Observando la matriz de componentes rotados (tabla 3) se puede confirmar que la tenencia de hijos menores o hijos mayores dependientes, el fallo de la sentencia (consensuado o no), la edad de los cónyuges y la CA (que marca la existencia de legislación propia o no en materia de asignación de la custodia) aparecen como variables con bastante importancia. Por tanto las variables hijos menores y mayores dependientes se enmarcarían en el primer componente, el fallo de la sentencia se enmarcarían dentro del segundo componente. En el tercer componente se enmarca la edad de los cónyuges mientras que en el cuarto y último se sitúa la CA.

La aparición de estas variables no hace más que constatar su importancia. La tenencia o no de hijos, ya sean menores o mayores dependientes, es bastante importante ya que el proceso de negociación puede ser más o menos complejo. 


\begin{tabular}{lcrrr}
\hline & \multicolumn{3}{c}{ Componente } \\
\cline { 2 - 5 } & 1 & 2 & 3 & 4 \\
\hline CCAA & 0,147 & $-0,032$ & $-0,141$ & $\mathbf{0 , 8 5 1}$ \\
Hijos menores de edad & $\mathbf{0 , 9 1 9}$ & 0,055 & 0,100 & $-0,003$ \\
Hijos mayores de edad dependientes & $\mathbf{0 , 9 0 1}$ & 0,007 & 0,108 & 0,005 \\
Edad 1er cónyuge & 0,101 & 0,007 & $\mathbf{0 , 7 9 2}$ & $-0,071$ \\
Nacionalidad 1er cónyuge & $-0,102$ & 0,015 & 0,055 & 0,050 \\
Edad 2do Cónyuge & 0,108 & 0,052 & $\mathbf{0 , 8 2 0}$ & 0,051 \\
Nacionalidad 2do cónyuge & $-0,281$ & 0,184 & 0,237 & 0,611 \\
Demandante del proceso & $-0,001$ & $-0,288$ & $-0,057$ & 0,033 \\
Fallo de la sentencia & 0,052 & $\mathbf{0 , 8 7 1}$ & 0,002 & 0,105 \\
Método de extracción: análisis de componentes principales. & & & \\
Método de rotación: Varimax con normalización Kaiser. & & &
\end{tabular}

Fuente: elaboración propia a partir de datos de la Estadística de Nulidades, Separaciones y Divorcios (2017).

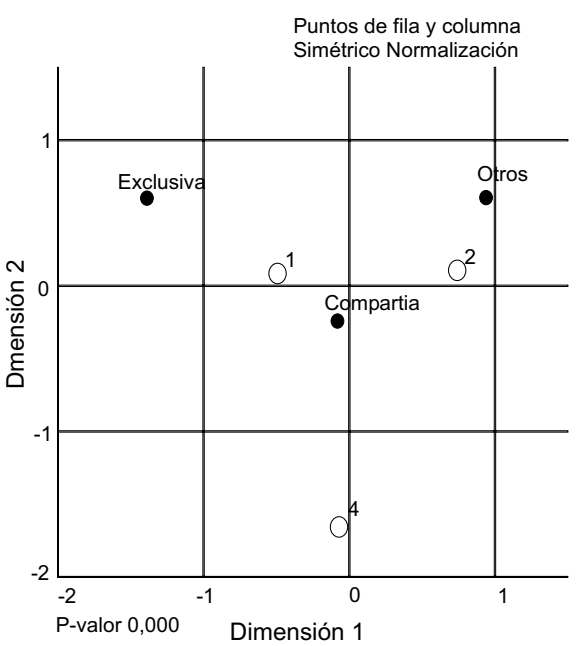

- Modalidad de custodia asignada

$\mathrm{N} .{ }^{\circ}$ hijos menores

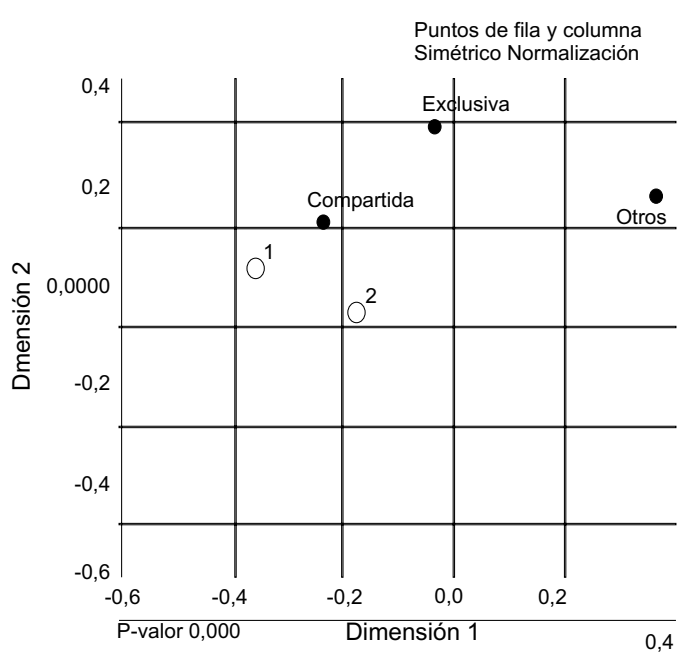

- Modalidad de custodia asignada

$\mathrm{N} .{ }^{\circ}$ hijos mayores dependientes

Figura 5 Análisis de correspondencias. Tipo de custodia según el número de hijos menores y mayores dependientes tras el divorcio de matrimonios del mismo sexo (2017)

Fuente: elaboración propia a partir de datos de la Estadística de Nulidades, Separaciones y Divorcios.

Ahondando en el proceso de asignación de la custodia tras el divorcio homosexual, parece que la custodia compartida es más probable en aquellos casos en los que se da la presencia de pocos hijos (figura 5). Concretamente hasta dos en el caso de que sean menores o mayores dependientes. Esto puede deberse a que el número 
de hijos que, de media, tienen los matrimonios compuestos por personas del mismo sexo es reducido. En este sentido se observan resultados de investigaciones, concretamente dos, contrapuestos pero que enfatizan la importancia de los hijos y el número de estos. Mientras que Maccoby y Mnookin (1992) indican que la custodia compartida es más probable en parejas con un solo hijo, Cancian y Meyer (1998) señalan que en el hipotético caso de que existan varios hijos tras del proceso de divorcio, los padres están de acuerdo en no separar a los hermanos en el $97 \%$ casos.

Estos mismos autores mantienen posiciones contrapuestas en relación a si esos hijos son menores o mayores dependientes. Cancian y Meyer (1998) no observan ninguna relación determinante con la edad de los niños mientras que Maccoby y Mnookin (1992) aseveran que los niños con edades comprendidas entre 2 y 9 años de edad, tienen más probabilidades de tener un régimen de custodia compartida que exclusiva. Para el caso de España, la base datos, no recoge la edad de los hijos por lo que no puede conocerse su determinación. No obstante, los resultados indican que los hijos menores y el número de estos tienen más importancia que en el caso de los mayores dependientes. Este hecho podría ser parte importante y diferencial de estos matrimonios.

Por otro lado, como se produzca el fallo de la sentencia parece también un factor determinante. Es de esperar que ante procesos consensuados las probabilidades de establecer la modalidad de custodia compartida aumenten en detrimento de la exclusiva. De esta forma, los resultados del análisis factorial indican que esta variable es clave para comprender qué tipo de custodia se asigna tras la ruptura matrimonial.

El grado de acuerdo en las disoluciones matrimoniales de parejas del mismo sexo es bastante alto (ver tabla 4), aumentando ligeramente desde el inicio del registro de los datos. Los niveles se mantienen a lo largo de la serie, prácticamente, por encima del $85 \%$. Indicar que el grado de consenso en este tipo de rupturas matrimoniales supera, de forma clara, el existente en disoluciones de matrimonios heterosexuales (en 2017 los procesos consensuados representan un 77,7\% del total). Esto, coincide con lo obtenido por Becerril y Jiménez (2016), que observaron como en este tipo de uniones, según se haya producido la ruptura (consensuada o contenciosa), la custodia de los hijos puede variar. También concuerda con lo comprobado por Wilcox, Wolchik y Braver (1998), donde parece que la custodia compartida es más probable cuando se experimentan baja conflictividad, o mayor consenso, entre los miembros de la pareja.

La edad de los cónyuges, enmarcada en el tercer componente, es igualmente fundamental. Estos matrimonios se caracterizan por tener una mayor diferencia etaria entre sus miembros que, por ejemplo, los matrimonios heterosexuales. En los matrimonios conformados por varones, de media, se da una mayor diferencia de edad entre ambos situada en 8 años mientras que las uniones entre mujeres registran una media de 5 años de diferencia. En este caso la media de edad al divorcio en matrimonios compuestos por varones es de 39 años mientras que la de los formados por mujeres desciende hasta los 36 . Esto puede suponer una característica esencial en la compresión de la importancia de esta variable. Jiménez y Becerril (2020) indican, para el caso de procesos de asignación de custodia en heterosexuales, que la 
Tipo de divorcio en matrimonios del mismo sexo (2008-2017) (\%)

\begin{tabular}{cccc}
\hline Año & Consensuado & No consensuado & Total \\
\hline 2008 & 82,8 & 17,2 & 100,0 \\
2009 & 84,9 & 15,1 & 100,0 \\
2010 & 81,1 & 18,9 & 100,0 \\
2011 & 83,2 & 16,8 & 100,0 \\
2012 & 85,5 & 14,5 & 100,0 \\
2013 & 85,2 & 14,8 & 100,0 \\
2014 & 86,0 & 14,0 & 100,0 \\
2015 & 86,6 & 13,4 & 100,0 \\
2016 & 87,0 & 13,6 & 100,0 \\
2017 & 86,8 & 13,3 & 100,0 \\
\hline
\end{tabular}

Fuente: elaboración propia a partir de datos de la Estadística de Nulidades, Separaciones y Divorcios.

tendencia general observada es que la probabilidad de que exista una custodia compartida comienza a ascender cuando alguno de los miembros de la pareja tiene más de 35 años.

Para la existencia de legislación propia, enmarcada en el cuarto componente, es de esperar que la disposición de regulaciones específicas en torno a la guardia y custodia de los hijos, que favorezcan la modalidad compartida, harán que esta se otorgue de manera más frecuente. Concretamente, una parte importante de las custodias compartidas que se otorgaron tras el divorcio de matrimonios de personas del mismo sexo (2017) se concentran en una CA con legislación propia como Cataluña (40\%) o Comunidad Valenciana (25\%). Este hecho ya sucede en el caso de los heterosexuales, dándose más casos de custodia compartida en aquellas CA con leyes propias.

Los resultados indican, de forma inequívoca, como estos componentes se relacionan en gran parte con la modalidad de custodia que se asigna tras la disolución de matrimonios del mismo sexo. Se concluye que la hipótesis II, "el fallo de la sentencia, la tenencia de hijos menores o mayores dependientes, la edad de los cónyuges y la CA influyen en la modalidad que se asigna", queda validada. Esto va en línea con lo concluido en estudios, a nivel nacional e internacional, que analizan las variables sociales que influyen en la asignación de la custodia tras la disolución de matrimonios heterosexuales.

Sin embargo variables como la nacionalidad de los cónyuges o el cónyuge demandante del proceso de divorcio no parecen estar tan estrechamente relacionadas con la modalidad de custodia finalmente otorgada, visto el valor obtenido tras la aplicación del análisis de interdependencia realizado. Que la nacionalidad no esté relacionada con la modalidad de custodia otorgada tras el divorcio de este tipo de matrimonios puede encontrar su motivación en que los matrimonios mixtos, o en los que ambos cónyuges no poseen nacionalidad española, suponen alrededor del $30 \%$ de los divorcios que se produjeron en 2017. Esto hace que aquellos casos en los que haya que dirimir procesos de asignación de custodia sean, prácticamente, residuales lo que conduce a que esta variable no sea hasta el momento tan importante como las mencionadas anteriormente. 


\section{Conclusiones}

El presente artículo aborda la asignación de la custodia tras la disolución de matrimonios compuestos por personas del mismo sexo y las variables sociales que influyen en la misma. Concretamente se atiende a la existencia de hijos menores, hijos mayores dependientes, la existencia de ley propia por comunidad autónoma, el fallo de la sentencia y la nacionalidad de los cónyuges. El interés principal de esta investigación reside en que, si bien la asignación de la custodia de los hijos/as tras la disolución de matrimonios heterosexuales, y muchos aspectos en torno a ella, han sido objeto de estudio recurrente por diferentes disciplinas tanto a nivel nacional como internacional, en España no se encuentran análisis alrededor de esta cuestión para el caso de los matrimonios del mismo sexo. Es preciso destacar que en este caso, aunque la población con la que se trabaja no es muy amplia, son todos los casos existentes en España desde la legalización del matrimonio homosexual hasta la actualidad.

La evolución de las disoluciones de este tipo de matrimonio ha ido variando a lo largo del tiempo. En un primer momento, las disoluciones de matrimonios conformados por varones fueron bastantes más altas que las de las mujeres. Más tarde, ambas aumentan de forma parecida, para después observar que las disoluciones entre varones sufren un ascenso vertiginoso (en 2016) en comparación con las producidas en matrimonios conformados por mujeres.

En cuanto a estas rupturas, el grado de acuerdo en las disoluciones es bastante alto, hecho que ha ido aumentando ligeramente desde el inicio del registro de los datos. Atendiendo a cada tipo de matrimonio, no se observan diferencias, siendo en ambos alto. Este hecho es algo que Goldberg y Allen (2013) ya mostraron es su investigación, siendo algo bastante importante en relación con la asignación de la custodia ya que, según Wilcox, Wolchik y Braver (1998), la custodia compartida es más probable cuando se experimentan baja conflictividad entre los miembros de la pareja. En este caso, los niveles de conflictividad son mínimos.

En cuanto a la tenencia de hijos, ya sean menores o mayores dependientes y el número de estos, se puede concluir que una parte importante de los matrimonios del mismo sexo que se divorcian lo hacen con la presencia de estos. Especificando aún más, aquellos matrimonios que se diluyen con la presencia de hijos suponen un porcentaje importante $(39,5)$. Desde el inicio de la serie histórica hasta el año 2017 , se ha producido un incremento del $13,5 \%$ en la cantidad de matrimonios entre personas del mismo sexo que se divorcian con hijos e hijas. Es necesario precisar que al analizar los divorcios homosexuales con presencia de hijos la población no es tan amplia como la de heterosexuales. En este sentido se tiene que especificar que el número de matrimonios totales desde 2005, o incluso año a año, distan mucho en cantidad. Sin embargo, en este caso no se está trabajando con una muestra seleccionada si no con la población completa.

Para la asignación de la custodia, se observa como la modalidad de custodia exclusiva ha ido disminuyendo, año a año, de forma progresiva a favor de la compartida. En 2012 la modalidad exclusiva suponía un 70\% de las custodias por un $30 \%$. Sin embargo, a partir de 2016 se produce un cambio de tendencia, pasando en 2017 la 
custodia compartida (51,5\%) por delante de la exclusiva (48,5\%). Esta tendencia al alza de la modalidad compartida es un hecho que se ha ido dando de forma generalizada en los procesos de asignación de la custodia. Aun así los matrimonios heterosexuales están muy alejados de los del mismo sexo en cuanto a custodia compartida. Para estos últimos la custodia compartida representa un 30,3\% en 2017.

Aspecto derivado es conocer las que variables influyen en la modalidad de custodia que se asigna tras la disolución de los matrimonios formados por personas del mismo sexo. Se ha podido comprobar que las variables aplicadas no hacen más que constatar su importancia e influencia, algo que ha sido vastamente comprobado en el caso de disoluciones heterosexuales. La tenencia de hijos menores o mayores dependientes, el fallo de la sentencia, la edad de los cónyuges, y la existencia de ley propia aparecen como variables relacionadas con el por qué se puede asignar un tipo u otro de custodia.

Ciertamente, es de esperar que ante procesos consensuados las probabilidades de establecer la modalidad de custodia compartida aumenten en detrimento de la exclusiva. En este caso, los resultados apuntan en esta dirección, indicando que esta variable es necesaria y clave para comprender qué tipo de custodia se asigna tras la ruptura matrimonial. Esta conclusión se encuentra en la línea de lo indicado por Wilcox, Wolchik y Braver (1998), donde parece que la custodia compartida es más probable cuando se experimentan baja conflictividad. Igualmente Becerril y Jiménez (2016) observaron como en los matrimonios del mismo sexo, según se haya producido la ruptura (consensuada o contenciosa), la custodia de los hijos puede variar.

En cuanto a la tenencia de hijos menores o mayores dependientes y el número de estos, los resultados del análisis apuntan a su alta importancia. Concretamente la existencia de hijos menores parece ser más decisiva a la de hijos mayores dependientes, ya que queda encuadrada en el primer factor. La importancia que se asigna a los hijos mayores o menores dependientes es algo que ha sido señalado, varias veces, para el caso de parejas heterosexuales (Jiménez y Becerril, 2020; Cancian y Meyer, 1998; Maccoby y Mnookin, 1992). Aun así, la base de datos utilizada presenta un gran hándicap con respecto al análisis de esta variable: no es posible conocer la edad de los hijos e hijas, limitando el conocimiento en profundidad de la misma y no permitiendo realizar una comparativa con estudios existentes a nivel internacional.

En relación a la existencia de legislación propia, es de esperar que en aquellas CA donde existe regulación propia y específica en este ámbito la custodia compartida se dé de forma más frecuente que en las que no existe. En este sentido, Solsona, Spijker y Ajenjo (2017) comprobaron para rupturas heterosexuales que una ley específica favorece el pacto entre los cónyuges respecto al cuidado de los hijos tras el divorcio, desde luego favorece la coparentalidad después de la ruptura. Por último, la edad los miembros del matrimonio divorciado es también importante. En los matrimonios del mismo sexo las diferencias etarias suelen ser mayores que en el caso de los heterosexuales, lo que puede dar lugar a que esta variable sea importante ya que cuando la edad de, al menos, uno de los cónyuges supera los 35 años se relaciona en mayor medida con la custodia compartida (Jiménez y Becerril, 2020). La diferencia presente en los matrimonios de personas del mismo sexo podría contribuir a este hecho. Por otro lado, variables como la nacionalidad o el cónyuge que 
demandante del divorcio no parecen estar intensamente relacionados con la modalidad de custodia que se otorga.

Puede concluirse que este conjunto de variables tienen peso e importancia en la modalidad de custodia que se asigna tras el divorcio de matrimonios del mismo sexo en España. Si bien no existen estudios en este ámbito, la presencia de los mismos en el caso de disoluciones matrimoniales ha permitido averiguar qué variables podían ser importantes y extrapolarlas a las disoluciones de matrimonios del mismo sexo. Se puede destacar la importancia de la existencia de consenso o disenso, ya que en los divorcios de matrimonios del mismo sexo la mayoría de procesos son consensuados. Esto conduce a lo concluido por Goldberg y Allen (2013) y Gartrell et al. (2011): la mayor parte de las custodias, cuando hay hijos, son compartidas debido a la relación entre baja conflictividad con mayores opciones de establecer la custodia compartida. Aun así, esto supone un primer acercamiento al análisis de la asignación de la custodia en este tipo de matrimonios desde un punto de vista sociológico en España.

Precisar que se encuentran algunas limitaciones en el estudio realizado. La serie de datos disponible puede resultar no muy extensa, abarcando el periodo temporal 2012-2017, debido a que hasta 2012 no se incluían datos referentes a las custodias para este tipo de matrimonios. Por otro lado el conjunto de variables disponibles en la ENSD es muy limitado. Aspectos como el nivel de ingresos y educativo de los cónyuges o la edad de hijos e hijas ayudarían a profundizar en el análisis y ofrecer resultados más completos. Otras características, como el papel que de los juzgados y equipos psicosociales, son también merecedoras de estudio. Incluir una vía de investigación cualitativa permitiría, de la misma forma, ofrecer mayor, y más profundo, conocimiento acerca de este fenómeno.

Por otro lado se está presente ante las primeras generaciones, pioneras en este caso, por lo que ciertamente el seguimiento a esta cuestión se convierte en necesario para conocer su evolución. En último lugar, la no disponibilidad de la edad de los hijos no permite ahondar en una cuestión que parece clave para entender la asignación de las custodias tras el divorcio de estos matrimonios.

\section{Bibliografía}

Andersson, Gunnar, Turid Noack, Ane Seierstad, y Harald Weedon-Fekjær (2006), “The demographics of same-sex marriages in Norway and Sweden", Demography, 43, pp. 79-98.

Bartholomew, David (1980), "Factor analysis for categorical data", Journal of the Royal Statistical Society, series B (Methodological), 42 (3), pp. 293-321.

Becerril, Diego (2008), "La percepción social del divorcio en España”, Revista Española de Investigaciones Sociológicas (REIS), 123, pp. 187-208.

Becerril, Diego (2014), “La custodia en los procesos de ruptura en España”, en Heriberto Cairo y Lucila Finkel (orgs.), Actas del XI Congreso de Español de Sociología. Crisis y Cambio. Propuestas desde la Sociología, Madrid, Federación Española de Sociología, pp. 174-184. 
Becerril, Diego (2015), “La ruptura matrimonial en España”, en Cristobal Torres. (org.), España 2015. Situación Social, Madrid, Centro de Investigaciones Sociológicas.

Becerril, Diego, y José Jiménez (2016), “Acuerdos y desacuerdos: matrimonios y rupturas en parejas homo y heterosexuales", en Diego Becerril y Antonio Lozano (orgs.), Sociología del Conflicto en las Sociedades Contemporáneas, Madrid, Dykinson, pp. 41-58.

Black, Dan, Gary Gates, Seth Sanders, y Taylor Lowell (2000), “Demographics of the gay and lesbian population in the United States: evidence from available systematic", Date Sources. Demography, 39 (2), pp. 139-154.

Black, Dan, Gary Gates, Seth Sanders, y Taylor Lowell (2002), “Why do gay men live in San Francisco?", Journal of Urban Economics, 51, pp. 54-76.

Blumstein, Philip, y Pepper Schwartz (1983), American Couples, Nueva York, Morrow.

Calvo, Kerman (2010), “Reconocimiento, ciudadanía y políticas públicas hacia las uniones homosexuales en Europa", Revista Española de Investigaciones Sociológicas, 129, pp. 37-59.

Cancian, María, y Daniel Meyer, (1998), “Who gets custody?”, Demography, 35, pp. 147-157.

Capote, Alberto, y José Nieto (2018), “Análisis geográfico del matrimonio entre personas del mismo sexo en España: evolución, distribución y perfiles tras una década de legalidad", Boletín de la Asociación de Geógrafos Españoles, 77, pp. 368-397.

Cortina, Clara (2016), "Demografía de las parejas homosexuales en España", Revista Española de Investigaciones Sociológicas, 153, pp. 3-22.

Cortina, Clara, y Anna Cabré (2010), “Las uniones homosexuales en España: una caracterización sociodemográfica a partir del censo de 2001", Papers, 3, pp. 565-583.

Déchaux, Jean (2009), Sociologie de la Famille, París, La Découverte.

De la Fuente, Santiago (2011), "Análisis de componentes principales", disponible en: https://bit.ly/3e623N8 (última cita en octubre 2021).

Esteve, Albert, Clara Cortina, y Anna Cabré (2009), “Long term trends in marital age homogamy patterns: Spain, 1922-2006", Population, 64 (1), pp. 173-202.

Fernández, Ana (2006), “La evolución jurídica del sistema matrimonial español desde la constitución de 1978 a la admisión del matrimonio homosexual”, Nueva Época, 3, pp. 93-112.

Ferrán, Magdalena (1996), SPSS para Windows 95. Programación y Análisis Estadístico, Madrid, McGraw-Hill.

Festy, Patrick, Marie Digoix, y Benedicte Garnier (2004), “What if same-sex couples exist in France after all?", en Patrick Festy y Marie Digoix (orgs.), Same-Sex Couples, Same-Sex Partnerships E Homosexual Marriages. A Focus on Cross-National Differentials, París, INED, pp. 193-209.

Finch, Janet (2007), “Visualización de familias”, Sociología, 41 (1), pp. 65-81.

Flaquer, Luis (2012), “Liberalización sin protección: un balance de los resultados de la aplicación de la Ley de divorcio de 2005", en Teresa Picontó (org.), La Custodia Compartida a Debate, Madrid, Dykinson, pp. 23-44.

Gato, Jorge, Daniela Freitas, y María Fontaine (2012), “Atitudes relativamente à homoparentalidade de futuros/as intervenientes da rede social", Psicología, 26 (1), pp. 71-95. 
Gato, Jorge, Margarida Rangel, y Daniela Leal (2020), “Adopción por mujeres lesbianas y hombres gay: desafíos percibidos y necesidades de formación para profesionales en Portugal", Adoption Quarterly, DOI: https://doi.org/10.1080/10926755.2020.1834044

Gartrell, Nanette, Bos Henny, Heidi Peyser, Amalia Deck, y Carla Rodas (2011),“Family characteristics, custody arrangements, and adolescent psychological wellbeing after lesbian mothers break up", Family Relations, 5, pp. 572-585.

Gimeno, Beatriz, y Violeta Barrientos (2009), “La institución matrimonial después del matrimonio homosexual", Iconos, Revista de Ciencias Sociales, 35, pp. 19-30.

Giraud, Colin (2013), "Le village gai de Montréal: une aventure urbaine minoritaire", Espaces et Sociétés, 154, pp. 33-48.

Goldberg, Abbie, y Katherine Allen (2013), “Same-sex relationship dissolution and LGB stepfamily formation: perspectives of young adults with LGB parents", Family Relations, 5, pp. 529-544.

Hoffman, Agustín, Juliana Stover, Guadalupe de la Iglesia, y Mercedes Fernández, (2013), “Correlaciones policóricas y tetracóricas en estudios factoriales exploratorios y confirmatorios", Ciencias Psicológicas, 7 (2), pp. 151-164.

Jiménez, José, y Diego Becerril (2020), “Main characteristics associated with the assignment of custodies after the divorce", Journal of Divorce E Remarriage, 61 (8), pp. 615-635.

Jiménez, José, Diego Becerril, y José García (2020), “La relación entre reformas legales y la asignación de la custodia compartida en España (2007-2017)", Revista Española de Ciencia Política, 53, pp. 119-142.

Jöreskog, Karl (2001), Analysis of Ordinal Variables 2. Cross-Sectional Data. Structural Equation Modelling with Lisrel 8.51, Jena, Friedrich Schiller Universitat.

Juárez, Miguel (2007), “La familia: algunos cambios sociales significativos", Miscelánea Comillas, 65 (27), pp. 815-826.

Juby, Heather, Celine Le Bourdais, y Nicole Marcil-Gratton (2005), “Sharing roles, sharing custody? Couples characteristics and children's living arrangements at separation", Journal of Marriage and Family, 67 (1), pp. 157-172.

Kubinger, Klaus (2003), “On artificial results due to using factor analysis for dichotomous variables", Psychology Science, 45 (1), pp. 106-110.

Kurdek, Lawrence (1991), "The dissolution of gay and lesbian couples", Journal of Social and Personal Relationship, 2, pp. 265-278.

Kurdek, Lawrence (1992), "Relationship stability and relationship satisfation in cohabiting gay and lesbian couples: a prospective longitudinal test of the contextual and interdependence model", Journal of Social and Personal Relationships, 1, pp. 125-142.

Leroy, Stéphane (2006), “Le Paris gay: éléments pour une géographie de l'homosexualité", Annales de Géographie, 646, pp. 579-601.

Maccoby, Eleanor, y Robert Mnookin (1992), Dividing the Child. Social and Legal Dilemmas of Custody, Cambridge, MA, Harvard University Press.

MacDowell, Cecilia, Ana Santos, Madalena Duarte, y Teresa Maneca (2009),

“Homoparen tality y desafíos a la ley: el caso Silva Mouta en la justicia portuguesa y el Tribunal Europeo de Derechos Humanos", Revista Crítica de Ciências Sociais, 87, pp. 43-68. 
Martín, María (2016), “Los derechos de las parejas del mismo sexo en Europa: estudio comparado", Revista Española de Derecho Constitucional, 107, pp. 219-253.

Meil, Gerardo (2001), “Nuevas formas de pareja: las parejas del mismo sexo", Abaco: Revista de Cultura y Ciencias Sociales, 29 (30), pp. 71-78.

Meil, Gerardo (2003), Las Uniones de Hecho en España, Madrid, CIS.

Montoya, Omar (2007), "Aplicación del análisis factorial a la investigación de mercados. Caso de estudio", Scentia et technica, 1 (35), pp 281-286.

Morgan, David (2011), Repensando las Prácticas Familiares, Basingstoke, Palgrave Macmillan.

Pichardo, José (2004), “Same-sex couples in Spain: historical, contextual and symbolic factors", en Marie Digoix y Patrick Festy (orgs.), Same-Sex Couples, Same-Sex Partnerships and Homosexual Marriages. A Focus on Cross-National Differentials. (col. Documents de Travail, n. ${ }^{\circ}$ 124), París, INED, pp. 159-173.

Remiggi, Frank (1998), “Le village gai de Montréal: entre le ghetto et l'espace identitaire", en Irène Demczuk y Frank Remiggi (dirs.), Sortir de l'Ombre, Montreal, VLB Editeur.

Rothblum, Esther, Kimberly Bálsamo, y Robert Wickham (2018), “Longitudinal predictors of relationship dissolution among same-sex and heterosexual couples", Couple and Family Psychology: Research and Practice, 6 (4), pp. 247-257.

Ruíz, Raúl, y Rafael Alcázar (2017), “Factores determinantes en la atribución de la custodia compartida: un estudio sociológico en los juzgados de familia", en Diego Becerril y Mar Venegas (orgs.), La Custodia Compartida en España, Madrid, Dykinson, pp. 109-124.

Sánchez, Pedro, y Marcos Bote (2009), "Familismo y cambio social: el caso de España”, Sociologías, 21, pp. 122-149.

Solsona, Montserrat, Jeroen Spijker, y Marc Ajenjo (2017), “Calidoscopio de la custodia compartida en las regiones de España", en Diego Becerril y Mar Venegas (orgs.), La Custodia Compartida en España, Madrid, Dykinson, pp. 45-72.

Suso, Anabel, Inés González, Ana Pérez, y María Velasco (2012), Análisis de los Modelos de Custodia Derivados de las Situaciones de Separación y Divorcio en España, Madrid, Ministerio de Sanidad, Servicios Sociales e Igualdad.

Wall, Karin, y Rita Gouveia (2014), "Cambiar los significados de la familia en las relaciones personales", Sociología Actual, 62 (3), pp. 352-373.

Wilcox, Kathryn, Sharlene Wolchik, y Sandford Braver (1998), "Predictors of maternal preference for joint or sole legal custody", Family Relations, 47 (1), pp. 93-101.

Widmer, Ed (2010), Configuraciones Familiares. Un Enfoque Estructural de la Diversidad Familiar, Londres, Ashgate.

Yeung, Jean, John Sandberg, Pamela Davis, y Sandra Hofferth (2001), “Children's time with fathers in intact families", Journal of Marriage and the Family, 63 (1), pp. 136-154.

Jose Manuel Jimenez Cabello. Profesor, Universidad de Granada, Granada, España. E-mail: josejicabello@ugr.es ORCID: 0000-0001-7518-2134. 
Diego Becerril Ruíz. Profesor, Universidad de Granada, Granada, España.

E-mail: Becerril@ugr.es ORCID: 0000-0002- 5876-8034

José Manuel García Moreno. Profesor, Universidad de Málaga, Málaga, España.

E-mail: jmgarciamoreno@uma.es ORCID: 0000-0003-2429-8764

Receção: 03 de março de 2020 Aprovação: 21 de abril de 2021 
